\section{ASPECTO SOCIODEMOGRÁFICO, CLÍNICO E QUALIDADE DE VIDA EM PACIENTES COM ARTRITE REUMATOIDE}

\author{
Sociodemographic clinical aspects and quality of life in \\ patients with rheumatoid arthritis \\ Aspecto sociodemográfico, clínico y calidad de vida de pacientes \\ con artritis reumatoide
}

\section{RESUMO}

Objetivo: Caracterizar os aspectos sociodemográficos, clínicos e a qualidade de vida (QV) de pacientes com artrite reumatoide (AR). Métodos: Tratou-se de um estudo transversal e descritivo, com abordagem quantitativa. Avaliou-se 22 indivíduos com AR, independentemente do sexo, e com idade superior a 18 anos. Os participantes foram encaminhados para o Laboratório de Análise Multissetorial da Universidade Estadual de Santa Catarina por médicos reumatologistas vinculados ao Sistema Único de Saúde, no período de junho a setembro de 2010. Utilizou-se uma ficha de avaliação para levantamento dos aspectos sociodemográficos e clínicos, nível de atividade da doença (Disease Activity Score 28) e o Questionário de Qualidade de Vida (36 Item Short-Form Health Survey). Resultados: Houve predomínio do sexo feminino, com $81,8 \%(n=18)$; etnia branca, com $90,9 \%(n=20)$; e da sexta década de vida, com 60,0 $( \pm 12,90)$ anos no sexo masculino. Dentre os sintomas, 17 (77,3\%) indivíduos referiram dor como a principal queixa. No nível de atividade da doença, verificou-se que os valores médios foram 4,4 $( \pm 1,56)$, com predomínio de $10(45,5 \%)$ indivíduos em atividade moderada. $\mathrm{Na} \mathrm{QV}$, evidenciou-se diferença significativa no domínio dor e aspecto social em função do nível de atividade da doença $(\mathrm{p}<0,05)$. No domínio dor, houve diferença significativa quando comparado os grupos alta e baixa atividade da doença $(p<0,01)$ e entre os grupos baixa e moderada $(p<0,01)$. Conclusão: Pôde-se identificar, nos indivíduos com artrite reumatoide participantes do estudo, prejuízo no seu estado geral de saúde e, além disso, o elevado nível de atividade da doença interferiu na sintomatologia dolorosa e comprometeu o aspecto social.

Descritores: Artrite Reumatoide; Qualidade de vida; Dor; Avaliação em Saúde; Condições Sociais.

\section{ABSTRACT}

Objective: To characterize the sociodemographic clinical aspects and quality of life of patients with rheumatoid arthritis (RA). Methods: This was a descriptive, cross-sectional quantitative research that evaluated 22 individuals with $R A$ over 18 years old, regardless of sex. Participants were referred to the Mulstisectoral Analysis Laboratory of the State University of Santa Catarina by rheumatologists of the Brazilian National Health System in the period from June to September 2010. It was used a form to obtain sociodemographic and clinical data, the level of disease activity (Disease Activity Score 28) and the Quality of Life Questionnaire (36 Item Short-Form Health Survey). Results: There was a prevalence of female gender, with 81.8\% ( $n=18)$; white ethnicity, with 90.9\% ( $n=20)$; and sixth decade of life, with 60 ( \pm 12.9$)$ years for male gender. Among the symptoms, 17 individuals (77.3\%) reported pain as the main complaint. Regarding the level of disease activity, the mean values were $4.47 \pm 1.56$ with a prevalence of 10 patients (45.5\%) presenting moderate activity. Concerning QoL, it was found a significant difference in the domains pain / social aspect and the level of disease activity $(p<0.05)$. Regarding the domain pain, there was a significant difference when comparing the groups high and low disease activity $(p<0.01)$ and between the low and moderate groups $(p<0.01)$. Conclusion: It was possible to identify in the individuals with RA participating in this study a deficit in their general state of health.
Artigo Original

1) Universidade do Estado de Santa Catarina - UDESC - Centro de Ciências da Saúde - CEFID - Florianópolis (SC) - Brasil

Recebido em: 18/03/2013

Revisado em: 16/08/2013 Aceito em: 11/09/2013 
Additionally, the high level of disease activity interfered with painful symptomatology and hindered social aspects.

Descriptors: Rheumatoid arthritis; Quality of life; Pain; Health Evaluation; Social Conditions.

\section{RESUMEN}

Objetivo: Caracterizar los aspectos sociodemográficos, clínicos y la calidad de vida $(\mathrm{CV})$ de pacientes con artritis reumatoide (AR). Método: Se trató de un estudio transversal $y$ descriptivo de abordaje cuantitativo. Se evaluó 22 individuos con AR independiente del sexo y de edad superior a 18 años. Los participantes fueron encaminados al Laboratorio de Análisis Multisectorial de la Universidad Provincial de Santa Catarina por médicos reumatólogos vinculados al Sistema Único de Salud en el periodo entre junio y septiembre de 2010. Se utilizó una ficha de evaluación para recogida de los aspectos sociodemográficos y clínicos, nivel de actividad de la enfermedad (Disease Activity Score 28) y el Cuestionario de Calidad de Vida (36 Item ShortForm Health Survey). Resultados: Hubo predominio del sexo femenino en el 81,8\% ( $n=18)$; etnia blanca en el 90,9\% $(n=20)$; $y$ de la sexta década de vida, con 60,0(土12,9) años en el sexo masculino. De los sintomas, 17 (77,3\%) individuos relataron dolor como queja principal. A nivel de actividad de la enfermedad se verifico que los valores medios fueron $4,47 \pm 1,56$, con predominio de $10(45,5 \%)$ individuos con actividad moderada. Respecto la CV se evidenció diferencia significativa en el dominio dolor y aspecto social en función del nivel de actividad de la enfermedad $(p<0,05)$. Hubo diferencia significativa en el dominio dolor al comparar los grupos alta y baja actividad de la enfermedad $(p<0,01)$ y entre los grupos baja y moderada actividad $(p<0,01)$. Conclusión: Se pudo identificar en los individuos con artritis reumatoide que participaron en el estudio, perjuicio del estado general de salud y además el nivel elevado de actividad de la enfermedad influyó en los sintomas de dolor y en el aspecto social.

Descriptores: Artritis Reumatoide; Calidad de Vida; Dolor; Evaluación en Salud; Condiciones Sociales.

\section{INTRODUÇÃO}

A artrite reumatoide (AR) é uma doença autoimune bastante complexa, caracterizada por sinovite proliferativa, gerando deformidade e destruição articular, em virtude da erosão óssea e cartilaginosa ${ }^{(1)}$. A prevalência da doença no Brasil varia em torno de $1 \%$ da população adulta e acomete principalmente o sexo feminino entre a quarta e sexta décadas de vida, com cerca de 2-3 mulheres para cada homem $^{(2)}$. Essa prevalência aumenta com o avanço da idade e mais de $60 \%$ dos indivíduos a partir dos 65 anos de idade são afetados. Estima-se que, por volta de 2030, quando o percentual da população nessa faixa etária deverá ser $20 \%$ maior, cerca de 67 milhões de indivíduos desse grupo serão afetados pela $\mathrm{AR}^{(3)}$.

A característica crônica da AR resulta no desenvolvimento de incapacidades físicas, com consequente diminuição das habilidades funcionais ${ }^{(4)}$. Sendo assim, a progressão da doença impõe dificuldades na realização das atividades da vida diária e profissional, refletindo negativamente nas condições econômicas para o indivíduo e para a sociedade.

Segundo os dados de internação hospitalar do DATASUS, no ano de 2012, foram registradas 19.249 internações devido à $\mathrm{AR}$ e outras poliartropatias inflamatórias, totalizando um gasto de R \$ 12.896.142,00, dos quais R $\$ 11.367 .555,00$ foram referentes a serviços hospitalares e R\$ 1.514.195,00, referentes a serviços profissionais ${ }^{(5)}$.

Por outro lado, também há um impacto evidente da AR nas condições físicas, psicológicas e sociais, bem como na qualidade de vida (QV) dos indivíduos afetados ${ }^{(6,7)}$. Nesse sentido, é importante entender e monitorar os efeitos da AR na saúde dos pacientes, uma vez que essas informações são fundamentais no estabelecimento do diagnóstico, do grau de atividade da doença e da eficácia terapêutica ${ }^{(8)}$.

Algumas pesquisas apresentaram as características desses pacientes baseadas na avaliação de diferentes aspectos da doença, como a funcionalidade, a impressão clínica, a QV, entre outros ${ }^{(1,9,10)}$. Entretanto, a AR apresenta particularidades bastante variáveis conforme a população acometida. Além disso, o curso da doença e os desfechos clínicos variam muito entre os pacientes. Dessa forma, as características da doença, assim como as variáveis sociodemográficas, estão recebendo cada vez mais atenção como fatores relevantes no prognóstico da $\mathrm{AR}^{(11)}$ e nas repercussões delas na saúde pública.

Diante do exposto, o objetivo do presente estudo foi caracterizar os aspectos sociodemográficos, clínicos e a QV de pacientes com AR, participantes de um projeto de pesquisa e extensão desenvolvido no Laboratório de Análise Multissetorial (MULTILAB) da Universidade Estadual de Santa Catarina (UDESC).

\section{MÉTODOS}

Tratou-se de um estudo transversal e descritivo, com abordagem quantitativa, realizado na cidade de Florianópolis-SC, durante o período de junho a setembro de 2010.

Participaram 22 indivíduos com diagnóstico de AR, selecionados através de amostragem por conveniência, independentemente do sexo, encaminhados por médicos reumatologistas da cidade de Florianópolis para um projeto 
de pesquisa e extensão desenvolvido no MULTILAB da UDESC.

Incluíram-se indivíduos que obedeceram aos critérios de classificação da American College of Rheumatology ${ }^{(12)}$ : adultos (idade superior a 18 anos), com artrite ativa, ou seja, um escore superior a 2,6 no Disease Activity Score (DAS-28) ${ }^{(13)}$, com acuidade visual preservada e capacidade de assinar o Termo de Consentimento Livre e Esclarecido. Foram adotados como critérios de exclusão: processo viral e/ou bacteriano inferior a 15 dias, gestantes, outras doenças autoimunes, deficiência cognitiva, história recente de lesões e/ou cirurgias ortopédicas.

Inicialmente, aplicou-se uma ficha de avaliação para coletar os aspectos sociodemográficos dos pacientes (idade, sexo, estado civil, etnia) e a história clínica da doença (queixa principal, tempo de diagnóstico, comorbidades e medicamentos em uso).

A determinação do nível de atividade da doença foi realizada com base no DAS-28, avaliando 28 articulações (ombros, cotovelos, punhos, metacarpofalangeanas, interfalangeanas proximais e joelhos, bilateralmente), contando o número de articulações doloridas (sem ponderar a intensidade da dor) e edemaciadas. Considerou-se dolorida quando a articulação apresentasse algum grau de desconforto, não necessariamente dor intensa, na avaliação feita por dígito-pressão de sua interlinha ou por sua mobilização passiva. Adicionalmente, foi obtida uma nota da percepção do paciente em relação à atividade da global da AR nos últimos 7 dias, por meio de uma escala visual analógica (escala $0-100$ ). Somado aos parâmetros anteriores, utilizou-se o valor da Proteína C-Reativa (PCR) para a realização de um cálculo que fornece o escore final do nível de atividade da doença ${ }^{(14)}$. Esse resultado pode variar de 0 a 10 , e quanto maior o valor, maior o nível de atividade da doença ${ }^{(15)}$. Os pacientes foram classificados de acordo com os seguintes níveis de atividade da doença: baixa (DAS- $28 \leq 3,2$ ), moderada (DAS- $28>3,2 \leq 5,1$ ) e alta (DAS-28 $>5,1)^{(14,15)}$.

A análise da PCR foi feita por meio de ensaios turbidimétricos, utilizando kit de reagentes destinado à determinação de PCR no soro (Bioténica), com leitura em espectrofotômetro a $540 \mathrm{~nm}$ (CONCEPT Bioplus 2000 - Biotécnica). Os resultados foram expressos em $\mathrm{mg} / \mathrm{L} \mathrm{de}$ sangue, sendo considerados normais valores até $6 \mathrm{mg} / \mathrm{L}$. Esses resultados foram utilizados para o cálculo do DAS28, conforme descrito anteriormente.

A avaliação da QV foi obtida por meio do questionário "36 Item Short-Form Health Survey" (SF-36), desenvolvido para avaliar, de forma genérica, a saúde física e mental ${ }^{(16)}$. O SF-36 possui tradução para língua portuguesa e foi validado no Brasil para pacientes com AR, sendo amplamente usado, uma vez que apresenta validade e confiabilidade ${ }^{(17,18)}$. Esse instrumento contém 36 questões acerca da saúde e bemestar, subdivididas em oito diferentes domínios: função física, limitação funcional decorrente da saúde física, função social, vitalidade, dores corporais, saúde mental, limitação funcional por causa de problemas emocionais e percepções gerais da saúde. Essa escala apresenta um escore de 0 (zero) a 100 (cem), em que zero corresponde ao pior aspecto de saúde e cem, ao melhor aspecto de saúde do indivíduo ${ }^{(18)}$.

Neste estudo, a distribuição de frequência dos escores obtidos para cada domínio avaliado no questionário SF-36 foi estratificada nos seguintes intervalos: 0-24,99; 25-49,99; 50-74,99 e 75-100.

Utilizou-se o programa SPSS versão 20.0 para a análise estatística descritiva e inferencial dos dados. As variáveis categóricas foram apresentadas através dos valores de frequência absoluta (n) e relativa (\%), e as variáveis numéricas, através dos valores de média e desvio padrão. Para comparar os domínios do SF-36 em função do grau da atividade da doença (baixa, modera e alta), utilizou-se o teste de Kruskall Wallis, seguido do teste U Mann-Whitney. Adotou-se nível de significância de $5 \%(\mathrm{p}<0,05)$.

Este estudo foi desenvolvido em concordância com os padrões éticos, respeitando a Resolução 196/96 do Conselho Nacional de Saúde e com o devido consentimento livre e esclarecido das participantes, sendo aprovado pelo Comitê de Ética de Pesquisa em Seres Humanos da Universidade do Estado de Santa Catarina (CEPSH - UDESC), sob protocolo de $n^{0} 54 / 2008$.

\section{RESULTADOS}

As características sociodemográficas são apresentadas na Tabela I, evidenciando o predomínio de pacientes do sexo feminino, casados e de etnia branca. Identificouse, também, predomínio da sexta década de vida no sexo masculino - 60,0 $( \pm 12,90)$ anos - e da quinta década de vida no sexo feminino - $58,1( \pm 7,20)$ anos, tendo média geral de $59,0( \pm 10,05)$ anos.

O perfil clínico da amostra encontra-se representado na Tabela II. A dor representou a principal queixa clínica para $17(77,3 \%)$ pacientes. Sobre a variável "medicações", o uso de terapia combinada foi apontada por $16(72,7 \%)$ pacientes. Em resposta ao escore da atividade da doença (DAS-28), a média geral foi de $4,4( \pm 1,56)$. A estratificação dessa variável mostrou que $10(45,5 \%)$ pacientes encontravam-se dentro do nível moderado de atividade da doença. Quanto à presença de comorbidades, $5(22,7 \%)$ apresentaram diabetes do tipo II, $4(18,2 \%)$ apresentaram hipertensão arterial e $5(22,7)$ tinham comorbidades associadas, considerando a combinação de comprometimentos metabólico, cardiovascular, osteomuscular e psicológico. 
Tabela I - Características sociodemográficas dos pacientes com artrite reumatoide $(n=22)$, levantadas por meio da ficha de avaliação. Florianópolis-SC, 2010.

\begin{tabular}{lcc}
\hline Características & & Frequência (\%) \\
\hline \multirow{2}{*}{ Gênero } & Feminino & $18(81,8)$ \\
& Masculino & $4(18,2)$ \\
& & \\
Etnia & Branco & $20(90,9)$ \\
& Negro & $1(4,5)$ \\
& Pardo & $1(4,5)$ \\
Estado Civil & & \\
& Casado & $16(72,7)$ \\
& Solteiro & $4(18,2)$ \\
& Divorciado & $1(4,5)$ \\
\hline
\end{tabular}

\%: percentual.

Tabela II - Características clínicas dos pacientes com artrite reumatoide (n=22), levantadas por meio da ficha de avaliação. Florianópolis-SC, 2010.

\begin{tabular}{lcc}
\hline Características & & Frequência (\%) \\
\hline \multirow{2}{*}{ Queixa principal } & Dor & $17(77,3)$ \\
& Edema & $3(13,6)$ \\
& Rigidez articular & $2(9,1)$ \\
Medicações & Monoterapia & $4(18,2)$ \\
& Terapia combinada & $16(72,7)$ \\
& Não faz uso & $2(9,1)$ \\
DAS-28 & & \\
& Baixa $\leq 3.2$ & $5(22,7)$ \\
Moderada 3.2 a 5.1 & $10(45,5)$ \\
Comorbidades & Alta $>5.1$ & $7(31,8)$ \\
& & $5(22,7)$ \\
& Diabetes & $4(18,2)$ \\
& Hipertensão & $3(13,6)$ \\
& Osteoporose & $1(4,5)$ \\
& Depressão & $5(22,7)$ \\
\hline
\end{tabular}

$\%$ : percentual. 
Verificou-se também que as médias do tempo de diagnóstico $(9,5 \pm 9,26$ anos) e do tempo de tratamento $(9,5$ $\pm 9,39$ anos) foram semelhantes.

Para verificar a repercussão da $\mathrm{AR}$ na $\mathrm{QV}$ desses pacientes, realizou-se uma análise descritiva do questionário SF-36, com base na distribuição de frequência percentual dos dados (Tabela III). Foi identificado maior frequência de indivíduos com escores abaixo de 50 para os domínios "dor" (68,2\%), "capacidade funcional" (54,5\%), "aspectos físicos" (72,7\%), "estado geral de saúde" (90,9\%) e "aspectos emocionais" $(59,1 \%)$, interferindo de forma negativa na QV deles. Por outro lado, verificou-se uma maior frequência de escores satisfatórios (acima de 50) nos domínios "vitalidade" (68,2\%), "saúde mental" $(72,7 \%)$ e "aspectos sociais" (59,1\%).

$\mathrm{Na}$ análise da influência do nível de atividade da doença (baixa, moderada e alta) sobre a qualidade de vida em relação aos domínios do SF-36, verificou-se diferença estatisticamente significativa nos domínios "dor" e "aspecto social" (Tabela IV). No domínio "dor", houve diferença significativa na comparação entre o grupo de alta atividade e os grupos de baixa $(p \leq 0,01)$ e moderada $(p \leq 0,001)$ atividade da doença. No domínio "aspecto social", foi verificada diferença significativa somente entre o grupo de alta $(\mathrm{p}=0,03)$ e moderada $(\mathrm{p}=0,03)$ atividade da doença.

Tabela III - Distribuição da frequência percentual nos diferentes domínios da qualidade de vida dos pacientes com artrite reumatoide (n=22). Florianópolis-SC, 2010.

\section{Frequência (\%)}

\section{Domínios da Qualidade de vida}

\begin{tabular}{cccc}
\hline \multicolumn{2}{c}{ Insatisfatório } & \multicolumn{2}{c}{ Satisfatório } \\
$\mathbf{0 - 2 4 , 9 9}$ & $\mathbf{2 5 - 4 9 , 9 9}$ & $\mathbf{5 0 - 7 4 , 9 9}$ & $\mathbf{7 5 - 1 0 0}$ \\
\hline $7(31,8)$ & $8(36,4)$ & $5(22,7)$ & $2(9,1)$ \\
$5(22,7)$ & $7(31,8)$ & $6(27,3)$ & $4(18,2)$ \\
$10(45,5)$ & $6(27,3)$ & $1(4,5)$ & $5(22,7)$ \\
$5(22,7)$ & $15(68,2)$ & $2(9,1)$ & $0(0)$ \\
$10(45,5)$ & $3(13,6)$ & $3(13,6)$ & $6(27,3)$ \\
$4(18,2)$ & $3(13,6)$ & $12(54,5)$ & $3(13,6)$ \\
$2(9,1)$ & $4(18,2)$ & $10(45,5)$ & $6(27,3)$ \\
$2(9,1)$ & $7(31,8)$ & $3(13,6)$ & $10(45,5)$ \\
\hline
\end{tabular}

Tabela IV - Comparação entre os grupos alta, moderada e baixa atividade da doença, nos diferentes domínios do questionário de qualidade de vida SF-36. Florianópolis-SC, 2010.

\begin{tabular}{lcccc}
\hline \multirow{2}{*}{ Domínios do SF-36 } & \multicolumn{3}{c}{$\begin{array}{c}\text { Atividade da doença } \\
\text { Média }( \pm \text { DP) }\end{array}$} & \multirow{2}{*}{$\boldsymbol{p}$} \\
\cline { 2 - 4 } & Alta & Moderada & Baixa & \\
\hline Dor & $22,85 \pm 9,13$ & $53,40 \pm 24,85$ & $49,6 \pm 16,83$ & $0,01^{*}$ \\
Capacidade Funcional & $37,85 \pm 27,21$ & $52,50 \pm 26,27$ & $40,0 \pm 16,95$ & 0,45 \\
Aspecto Físico & $10,71 \pm 13,36$ & $42,50 \pm 47,21$ & $35,0 \pm 37,91$ & 0,38 \\
Estado Geral de Saúde & $26,00 \pm 12,98$ & $36,90 \pm 13,44$ & $42,2 \pm 19,44$ & 0,09 \\
Aspecto Emocional & $14,27 \pm 26,20$ & $59,90 \pm 46,61$ & $39,98 \pm 43,45$ & 0,11 \\
Vitalidade & $42,14 \pm 29,41$ & $61,50 \pm 21,47$ & $43,0 \pm 16,04$ & 0,18 \\
Saúde Mental & $48,57 \pm 24,59$ & $70,00 \pm 19,16$ & $58,40 \pm 15,38$ & 0,12 \\
Aspecto Social & $40,78 \pm 27,12$ & $75,00 \pm 27,63$ & $42,50 \pm 28,77$ & $0,05^{\#}$ \\
\hline
\end{tabular}

DP: desvio padrão

* Significativo $\mathrm{p} \leq 0,05$, na comparação entre o grupo em alta atividade da doença em relação aos grupos baixa e moderada atividade. \#Significativo $\mathrm{p} \leq 0,05$, na comparação entre os grupos alta e moderada atividade da doença - Teste de Kruskall Wallis seguido do test $U$ de Mann-Whtiney. 


\section{DISCUSSÃO}

As características sociodemográficas de indivíduos com $\mathrm{AR}$, principalmente quanto ao sexo, à idade e à etnia, têm sido descritas na literatura ${ }^{(2,4,19)}$ e coincidem com os resultados do presente estudo. A caracterização dos indivíduos avaliados foi definida pela prevalência do sexo feminino, de etnia branca, entre a quinta e sexta década de vida e frequentemente casados.

A maior ocorrência da AR em mulheres já está bem definida na literatura ${ }^{(20)}$. Em estudo bibliográfico realizado com 26 teses a respeito da AR, observou-se prevalência do sexo feminino em todos os trabalhos ${ }^{(21)}$. Com relação à faixa etária, na maioria dos estudos, a média de idade varia entre 50,5 e $59,3 \operatorname{anos}^{(22,23)}$. Em pesquisa realizada com 5.235 sujeitos com AR, a média de idade encontrada foi de 57 anos $^{(24)}$. Quanto à etnia, um estudo de levantamentos demográficos evidenciou que $70 \%$ dos indivíduos com AR eram de origem caucasoide, $20 \%$ mulatos, $6 \%$ negros e $4 \%$ asiáticos $^{(19)}$.

Os aspectos clínicos avaliados no presente estudo também foram semelhantes aos dados da literatura. Com relação ao tempo médio de diagnóstico, verificou-se que foi de 9,59 $( \pm 9,26)$ anos. Algumas pesquisas ${ }^{(6,25)}$ também verificaram que a duração média da artrite reumatoide era de $9,8( \pm 7,2)$ anos e $9,79( \pm 7,6)$, respectivamente. Ressaltase ainda que o fato de o tempo de tratamento ter coincidido com o tempo de diagnóstico da AR tende a favorecer um mal prognóstico da doença, postergando seus efeitos crônicos.

Dentre os sintomas da AR, a dor foi indicada como a principal queixa. Em vários estudos, evidenciou-se essa sintomatologia como uma das principais manifestações iniciais, podendo acometer diversas regiões articulares, como articulação glenoumeral, coxofemoral, do joelho e pé $^{(1,2,6,20)}$.

Quanto ao tratamento daAR, verificou-se predominância da terapia combinada, de drogas antirreumáticas modificadoras da doença (DMARD) com anti-inflamatórios e/ou imunossupressores. Os resultados encontrados foram consistentes com estudo anterior ${ }^{(26)}$, que quantificou a polifarmácia em grupo de pacientes com AR e constatou o predomínio de combinações de drogas DMARD como principal esquema terapêutico. As DMARDS apresentam o potencial de reduzir ou evitar a lesão articular, preservar a integridade e a função da articulação, reduzir os custos aos cuidados de saúde e manter a produtividade econômica do paciente com $\mathrm{AR}^{(27)}$.

A acessibilidade ao tratamento com essas poderosas drogas geram maiores preocupações no sentido do alto custo das medicações que podem dificultar a implementação da recomendação da Liga Europeia ao Combate do Reumatismo (EULAR), a qual preconiza a utilização de drogas biológica e sintéticas no tratamento da AR logo que esta é diagnosticada ${ }^{(28)}$.

Os resultados do DAS-28 caracterizaram o predomínio dosindivíduosemmoderada atividade da doença $(4,47 \pm 1,56)$, corroborando com os achados de outras pesquisas ${ }^{(2,19,21)}$. Em pacientes de longa data de diagnóstico, a atividade da doença está relacionada com o comprometimento da $\mathrm{QV}^{(22)}$. Além disso, o nível de atividade da doença pode interferir no domínio saúde fisica do SF-36 ${ }^{(29)}$.

Além da $\mathrm{AR}$, os pacientes apresentaram outras doenças associadas ao comprometimento metabólico, ou sua combinação com desordens cardiovasculares, osteomusculares ou psicológicas. Apesar de os estudos sobre a prevalência de síndrome metabólica nos portadores de artrite ainda não apontarem conclusões definitivas, sua presença está diretamente associada com piores parâmetros prognósticos e de atividade de doença ${ }^{(30)}$, influenciando negativamente na saúde individual e coletiva.

Outra comorbidade importante detectada neste estudo foi as desordens cardiovasculares, que estão associadas com o aumento da mortalidade, devido principalmente a causas isquêmicas, como o infarto do miocárdio e a insuficiência cardíaca congestivaa ${ }^{(9,31)}$. Sabe-se que o uso de glicocorticoides em longo prazo está associado a uma alta prevalência de hipertensão $\operatorname{arterial}^{(32)}$ e hiperglicemia ${ }^{(33)}$, assim como o uso de anti-inflamatórios não esteroidais (AINES) pode exarcebar a pré-existência da hipertensão ${ }^{(34)}$.

A combinação desses dados descritos é capaz de influenciar na QV desses pacientes. Isso foi verificado na avaliação do questionário SF-36, no qual se evidenciou prejuízo para os domínios dor, capacidade funcional, aspectos físicos, estado geral de saúde e aspectos emocionais. Os domínios "vitalidade", "saúde mental" e "aspectos sociais" apresentaram resultados mais satisfatórios para a maioria dos indivíduos avaliados, embora tenha sido observada distribuição de frequência também nos escores mais baixos da escala.

Estudos confirmam que pacientes com AR apresentam comprometimento da QV quando comparado a indivíduos saudáveis ${ }^{(35)}$ Artrite apresenta um efeito significativo na qualidade de vida, no sentido de representar um perigo para a integridade física, psicológica, social, econômica e bemestar, privando muitas vezes as pessoas de sua liberdade e independência.

Em estudo realizado com 1.024 pacientes $^{(36)}$, constatou-se que a dor foi o domínio no qual mais de $70 \%$ dos pacientes com AR desejavam melhorar. Tal afirmativa converge para os resultados dessa pesquisa, na qual também houve prevalência da dor como queixa principal. Essa sintomatologia pode repercutir negativamente em outros domínios do SF-36, principalmente aqueles relacionados 
à capacidade funcional. Tanto os aspectos relacionados à dor quanto os relacionados às limitações físicas apresentam grande sensibilidade às alterações da $\mathrm{QV}^{(16,17)}$. $\mathrm{O}$ controle da dor e a prevenção de incapacidade são fundamentais para a QV e servem como medida para a redução da atividade da doença ${ }^{(37)}$.

Por fim, salienta-se que o número reduzido de indivíduos avaliados, visto que a seleção dos participantes ocorreu por conveniência, constitui uma limitação do presente estudo.

\section{CONCLUSÃO}

A caracterização dos pacientes com AR possibilitou identificar um maior acometimento no sexo feminino, preferencialmente de etnia branca e entre a quinta e a sexta década de vida. Além disso, a dor foi relatada como a principal queixa referente à doença.

Pôde-se identificar, nos indivíduos com artrite reumatoide, prejuízo no seu estado geral de saúde e, além disso, o elevado nível de atividade da doença interferiu na sintomatologia dolorosa e comprometeu o aspecto social.

\section{REFERÊNCIAS}

1. Aletaha D, Funovits J, Smolen JS. Physical disability in rheumatoid arthritis is associated with cartilage damage rather than bone destruction. Ann Rheum Dis. 2011;70(5):733-9.

2. Senna ER, Barros AL, Silva EO, Costa IF, Pereira LV, Ciconelli RM, et al. Prevalence of rheumatic diseases in Brazil: a study using the COPCORD approach. J Rheumatol. 2004;31(3):594-7.

3. Centers for Disease Control and Prevention. Projected state-specific increases in self-reported doctordiagnosed arthritis and arthritis-attributable activity limitations United States, 2005-2030. MMWR Morb Mortal Wkly Rep. 2007;56(17):423-5.

4. Michaud K, Llonch V, Oster G. Mortality risk by functional status and health-related quality of life in patients with rheumatoid arthritis. J Rheumatol. 2012;39(1):54-9.

5. Ministério da Saúde (BR), DATASUS. Sistema de informações hospitalares do SUS (SIH/SUS): morbidade hospitalar do SUS por local de internação, Brasil [acesso em 2013 Set 23]. Disponível em: http:// tabnet.datasus.gov.br/cgi/tabcgi.exe?sih/cnv/niuf.def.

6. Corbacho MI, Dapueto JJ. Assessing the functional status and quality of life of patients with rheumatoid arthritis. Rev Bras Reumatol. 2010;50(1):31-43.
7. Morris A, Yelin EH, Panopalis P, Julian L, Katz PP. Long-term patterns of depression and associations with health and function in a panel study of rheumatoid arthritis. J Health Psychol. 2011;16(4):667-77.

8. Mota LMH, Laurindo IMM, Santos Neto LL dos. Avaliação prospectiva da qualidade de vida em uma coorte de pacientes com artrite reumatoide inicial. Rev Bras Reumatol. 2010;50(3):249-61.

9. Ong KL, Wu BJ, Cheung BM, Barter PJ, Rye KA. Arthritis: its prevalence, risk factors, and association with cardiovascular diseases in the United States, 1999 to 2008. Ann Epidemiol. 2013;23(2):80-6.

10. Bianchi WA, Elias FR, Pinheiro GD, Gayer CR, Carneiro C, Grynzpan R, et al. Analysis of the association of fatigue with clinical and psychological variables in a series of 371 Brazilian patients with rheumatoid arthritis. Rev Bras Reumatol. 2014;54(3):200-7.

11. Carmona L, Cross M, Williams B, Lassere M, March L. Rheumatoid arthritis. Best Pract Res Clin Rheumatol. 2010;24(6):733-45.

12. Arnett FC, Edworthy SM, Bloch DA, Mcshane DJ, Fries JF, Cooper NS, et al. The American rheumatism association 1987 revised criteria for the classification of rheumatoid arthritis. Arthritis Rheum.1988;31(3):315-24.

13. Prevoo MLL, Vanthof MA, Kuper HH, Vanleeuwen MA, Vandeputte LBA, Vanriel P. Modified diseaseactivity scores that include 28-joint counts - development and validation in a prospective longitudinal-study of patients with rheumatoid-arthritis. Arthritis Rheum. 1995;38(1):44-8.

14. Fransen J, Riel PLCM. DAS remission cut points. Clin Exp Rheumatol. 2006;24 (Suppl 43):29-32.

15. Fransen J, Visser K, Van Dongen H, Huizinga T, Van Riel PCM, Van De Heijd DM. Validity of the disease activity score in undifferentiated arthritis. Arthritis Care Res. 2010;62(10):1392-8.

16. Picavet HS, Hoeymans N. Health related quality of life in multiple musculoskeletal diseases: SF-36 and EQ-5D in the DMC3 study. Ann Rheum Dis. 2004;63(6):7239.

17. Walker JG, Littlejohn GO. Measuring quality of life in rheumatic conditions. Clin Rheumatol. 2007;26(5):6713.

18. Ciconelli RM, Ferraz MB, Santos W, Meinão I, Quaresma MR. Tradução para a língua portuguesa e validação do questionário genérico de avaliação de 
qualidade de vida SF-36 (Brasil SF-36). Rev Bras Reumatol. 1999;39(3):143-50.

19. Louzada Júnior P, Souza BDB, Toledo RA, Ciconelli RM. Análise descritiva das características demográficas e clínicas de pacientes com artrite reumatóide no estado de São Paulo, Brasil. Rev Bras Reumatol. 2007;47(2):84-90.

20. Mont'Alverne ARS, Barbieri R, Montenegro RM, Anti SMA, Giorgi RDN, Chahade WH. Artrite Reumatoide no idoso: estudo de 35 casos. Geriatria Gerontol. 2011;5(3):159-62.

21. Abreu MM, Kowalski SC, Ciconelli RM, Ferraz MB. Avaliação do perfil sociodemográfico, clínicolaboratorial e terapêutico dos pacientes com artrite reumatóide que participaram de projetos de pesquisa na Escola Paulista de Medicina, nos últimos 25 anos. Rev Bras Reumatol. 2006;46(2):103-9.

22. Kojima M, Kojima T, Ishiguro N, Oguchi T, Oba M, Tsuchiya H, et al. Psychosocial factors, disease status, and quality of life in patients with rheumatoid arthritis. J Psychosom Res. 2009;67(5):425-31.

23. Barnabe C, Xiong J, Pope JE, Boire G, Hitchon C, Haraoui B, et al. Factors associated with time to diagnosis in early rheumatoid arthritis. Rheumatol Int. 2014;34(1):85-92.

24. Sokka T, Häkkinen A, Kautiainen $\mathrm{H}$, Maillefert JF, Toloza S, Hansen TM, et al. Physical inactivity in patients with rheumatoid arthritis: Data from twentyone countries in a cross-sectional, international study. Arthritis Rheum. 2008;59(1):42-50.

25. Azevedo AB, Ferraz MB, Ciconelli RM. Indirect costs of rheumatoid arthritis in Brazil. Value Health. 2008;11(5):869-77.

26. Bagatini F, Blatt CR, Maliska G, Trespash GV, Pereira IA, Zimmermann AF, et al. Potential drug interactions in patients with rheumatoid arthritis. Rev Bras Reumatol. 2011;51(1):20-39.

27. Mota LMH, Cruz BA, Brenol CV, Pereira IA, Rezende-Fronza LS, Bertolo MB, et al. Consenso 2012 da Sociedade Brasileira de Reumatologia para o tratamento da artrite reumatoide. Rev Bras Reumatol. 2012;52(2):135-74.

28. Putrik, P, Sokka T, Ramiro S, Boonen A. Impact of socioeconomic gradients within and between countries on health of patients with rheumatoid arthritis (RA): lessons from QUEST RA. Best Pract Res Clin Rheumatol. 2012;26(5):705-20.
29. Alishiri GH, Bayat N, Salimzadeh A, Salari A, Hosseini SM, Rahimzadeh S, et al. Health-related quality of life and disease activity in rheumatoid arthritis. J Res Med Sci. 2011;16(7):897-903.

30. Cunha VR, Brenol CV, Brenol JCT, Xavier RM. Artrite reumatoide e síndrome metabólica. Rev Bras Reumatol. 2011;51(3):260-8.

31. Soeiro AM, Haddad M, Almeida MCF, Ruppert AD, Serrano Junior AV. Artrite reumatoide e doença cardiovascular: o que sabemos e o que podemos fazer pelo paciente na atualidade? Rev Port Cardiol. 2012;31(3):225-32.

32. Panoulas VF, Douglas KMJ, Stavropoulos-Kalinoglou A, Metsios GS, Nightingale P, Kita MD, et al. Longterm exposure to medium-dose glucocorticoid therapy associates with hypertension in patients with rheumatoid arthritis. Rheumatology. 2008;47(1):72-5.

33. Kim SY, Yoo CG, Lee CT, Chung HS, Kim YW, Han SK, et al. Incidence and risk factors of steroid-induced diabetes in patients with respiratory disease. J Korean Med Sci. 2011;26(2):264-7.

34. Faselis C, Doumas M, Papademetriou V. Common secondary causes of resistant hypertension and rational for treatment. Int J Hypertens. 2011;2011:236-9.

35. Campos APR, Silva CM, Castro SS, Graminha CV. Depressão e qualidade de vida em indivíduos com artrite reumatoide e indivíduos com saúde estável: um estudo comparativo. Fisioter Pesqui. 2013;20(4):4017.

36. Heiberg T, Kvien TK. Preferences for improved health examined in 1,024 patients with rheumatoid arthritis: Pain has the highest priority. Arthritis Care Res. 2002; 47(4):391-7.

37. Cho SK, Kim D, Jun JB, Bae SC, Sung YK. Factors influencing quality of life (QOL) for Korean patients with rheumatoid arthritis (RA). Rheumatol Int. 2013;33(1):93-102.

\section{Endereço para correspondência:}

Monique da Silva Gevaerd

Universidade do Estado de Santa Catarina - UDESC

Centro de Ciências da Saúde - CEFID

Laboratório de Análises Multisetorial - MULTILAB

Rua Paschoal Simone, 358

Bairro: Coqueiros.

CEP: 88080-350 - Florianópolis - SC - Brasil

E-mail: moniquegevaerd@yahoo.com.br 